\title{
Indications and long-term results of enteral feeding in infancy and childhood
}

\author{
Danyah Mahmoud Alsafadi ${ }^{1 *}$, Gaida Sharaf Alzahrani², Qasem Mohammed Alhayek ${ }^{3}$, \\ Hawra Hassan Alghazwi ${ }^{4}$, Waleed Abdulwahab Alzahrani ${ }^{5}$, Reema Saeed AL Qahtani ${ }^{6}$, \\ Afnan Jaber Alfahhad ${ }^{7}$, Hanan Ahmed Hazzazi ${ }^{7}$, Bashayr Dimshaq Alyami ${ }^{8}$, \\ Nasser Ali Alsakhin', Raad Aidh Althagafi ${ }^{10}$
}

\author{
${ }^{1}$ Department of Pediatrics, Al Aziziyah Children Hospital, Jeddah, Saudi Arabia \\ ${ }^{2}$ College of Medicine, Umm Al-Qura University, Mecca, Saudi Arabia \\ ${ }^{3}$ Department of Orthopaedics, Houtat Bani Tamim General Hospital, Houtat Bani Tamim, Saudi Arabia \\ ${ }^{4}$ Department of Emergency Medicine, Abqaiq General Hospital, Abqaiq, Saudi Arabia \\ ${ }^{5}$ Department of Emergency Medicine, King Fahad General Hospital, Jeddah, Saudi Arabia \\ ${ }^{6}$ College of Medicine, Imam Mohammad Ibn Saud Islamic University, Riyadh, Saudi Arabia \\ ${ }^{7}$ Emergency Medical Services, Alrafee'ah General Hospital, AlRafee'ah, Saudi Arabia \\ ${ }^{8}$ Department of Radiology, King Khalid Hospital, Najran, Saudi Arabia \\ ${ }^{9}$ College of Medicine, Medical University of Lublin, Lublin, Poland \\ ${ }^{10}$ Primary Healthcare, Land Forces Medical Administration, Abha, Saudi Arabia
}

Received: 14 November 2021

Accepted: 29 November 2021

\section{*Correspondence:}

Dr. Danyah Mahmoud Alsafadi,

E-mail: dr_ds555@hotmail.com

Copyright: (C) the author(s), publisher and licensee Medip Academy. This is an open-access article distributed under the terms of the Creative Commons Attribution Non-Commercial License, which permits unrestricted non-commercial use, distribution, and reproduction in any medium, provided the original work is properly cited.

\begin{abstract}
Enteral feeding is more favorable than parenteral one because it can significantly intervene against the colonization of bacteria and preserve gut functions. However, this necessitates the presence of a good-functioning gastrointestinal tract. Young infants, critically ill children, and patients with neurological disabilities are the most probable candidates to perform enteral feeding. In the present literature review, we have discussed the indications and long-term results of enteral feeding in pediatric settings. Our results show that modality is a safe and efficacious modality in these settings with favorable outcomes and fewer adverse events and complications. Many indications were reported for the modality, and in general, children that usually suffer from severe weight deficit, weight faltering, and growth retardation are indicated to receive enteral nutrition. Some contraindications were also reported in the literature, and in general, conditions affecting the function and health status gastrointestinal tract should recommend against conducting approaches of enteral feeding. Different complications were reported, including mechanical, metabolic, infectious, gastrointestinal, and drug-related complications that might lead to worsened prognosis and can significantly impact the long-term outcomes of these patients. Therefore, paying adequate attention should be considered in these cases to prevent the development of these complications, and provide all the necessary procedures to potentially manage the expected adverse events.
\end{abstract}

Keywords: Enteral feeding, Pediatric, Gastrointestinal tract, Management, Nutrition, Supplementation

\section{INTRODUCTION}

Secondary to stunted growth or wasting, or following inadequate nutritional intake, evidence indicates the validity and effectiveness of enteral feeding as a nutritional supplementation that can increase growth rate in the affected patients. ${ }^{1,2}$ Enteral feeding is also more favorable than parenteral one because it can significantly 
intervene against the colonization of bacteria and preserve gut functions. However, this necessitates the presence of a good-functioning gastrointestinal tract. Young infants, critically ill children, and patients with neurological disabilities are the most probable candidates to perform enteral feeding. ${ }^{3}$

Many previous studies have reported the efficacy of enteral feeding for providing the required nutritional supplementation for high-risk children with stunted growth and other conditions. However, it should be noted that such approaches can be also relatively and absolutely contraindicated in some situations based on the health status of the patients and their gastrointestinal tract. ${ }^{3,4}$ Besides, some complications were also reported for the condition, and therefore, evidence indicates that the modality should not be considered unless strongly indicated. Otherwise, alternative management plans should be provided to adequately manage the potentially present adverse events. ${ }^{4,5}$ In the present literature review, we aim to investigate the indications and long-term outcomes of enteral feeding in pediatric settings.

\section{LITERATURE REVIEW}

This literature review is based on an extensive literature search in Medline, Cochrane, and EMBASE databases which was performed on $20^{\text {th }}$ of October 2021 using the medical subject headings (MeSH) or a combination of all possible related terms, according to the database. To avoid missing poetential studies, a further manual search for papers was done through Google Scholar, while the reference lists of the initially included papers. All relevant papers were screened for useful information, with no limitations posed on date, language, age of participants, or publication type.

\section{DISCUSSION}

Different considerations should be made to the method and indications of enteral feeding based on the age of the pediatric patient because the course and type of the nutrition course hugely vary in the pediatric population based on this factor. In general, the attending physicians should first manage any underlying condition or disease in combination with the enteral feeding process to enhance the quality of care in these patients. ${ }^{1,6}$ Furthermore, before deciding the appropriate enteral feeding plan, physicians should determine whether the condition of the patient is deteriorating from a specific disease or not, the presence of potential adverse event from the treatment modality, the presence of chronic or acute disorders, life expectancy, and current age of the pediatric patient that is indicated to receive enteral feeding. The function of the gastrointestinal tract, and whether the general condition of the affected patient is stable or reversible hugely determines the duration and approach of the enteral feeding process. ${ }^{7,8}$ Many indications have been proposed to conduct enteral feeding in the pediatric population, and these indications do not remarkably vary from the pre-specified indications in conditions requiring nutritional support. In general, the main indication of the modality is for children that usually suffer from severe weight deficit, weight faltering, and growth retardation. ${ }^{9,10}$ This is because the usually feeding approaches do not adequately need the nutritional and energy needs of these children, and therefore, enteral feeding is indicated in these situations to enhance the quality of feeding and meet the required amount of nutrition for these children. It has been furtherly indicated in the management course of certain pediatric diseases and conditions, as supportive therapeutic approaches, like food intolerance, food allergy, and Crohn's disease. ${ }^{11,12}$ However, it should be noted that enteral feeding can only be conducted in cases when the general condition of the gastrointestinal tract allows for conducting this approach. Otherwise it would be difficult to perform and can be more harmful to the general condition of the affected child. ${ }^{1,13}$ Evidence indicates that the modality can be used on various levels for the management, and even diagnosis, of the various conditions and disorders. However, clinicians in the different settings should consider the feasibility of the approach for the presenting patients and the cost considerations, especially for populations in the poor socio-economic communities. ${ }^{13,14}$ Necrotizing or premature enterocolitis are also reported indications for conducting enteral feeding supply in the affected children based on the severity and expected prognosis of the underlying condition. ${ }^{15}$ However, it should be noted that conducting enteral feeding is contraindicated in certain situations where the modality might be associated with more adverse events rather than the expected favorable outcomes. There are absolute and relative contraindications. Intestinal perforation or obstruction, mechanical or paralytic ileus, and other problems related to the gastrointestinal tracts of the affected children are absolute contraindications that indicate that the modality should not be conducted until dealing with these conditions. On the other hand, high-output enteric fistula, gastrointestinal bleeding, diffuse peritonitis, toxic megacolon, necrotizing enterocolitis, and intestinal dysmotility have been reported to be different relative contraindications factors. It should also be noted that minimum intestinal supply should be considered, and full fasting should not be approached before conducting the enteral feeding plan. ${ }^{16,17}$

Many outcomes have been reported for enteral feeding, including favorable and unfavorable events or complications. Different investigations that using enteral feeding is associated with man favorable outcomes. For instance, a previous investigation by Chen et al reported that enteral feeding was associated with maintaining adequate body growth, abrupt weaning of parenteral nutrition, and improvement in symptoms of the underlying conditions. It has been furtherly reported that no major complications were reported in the population that were indicated to receive enteral feeding, and therefore, this modality has been considered an 
efficacious, minimally-invasive, and safe procedure. Many studies have reported that the success rate of endoscopic-guided enteric feedings is very high, and can be up to $90 \% .^{18-20}$ Improvement in symptoms of the underlying conditions for which patients were indicated to receive enteral feeding was also reported to be remarkable after conducting this approach. In mechanically-ventilated adult patients, evidence indicates that the risk of aspiration pneumonia is significantly reduced following the process of small bowel feeding. ${ }^{21,22}$ However, evidence regarding these favorable events in the pediatric population has not been strengthened by adequate investigations in the literature. ${ }^{23,24}$ On the other hand, a previous study reported that reduced episodes of aspiration pneumonia were significantly associated with the administration of enteral feeding in children and infants. ${ }^{25}$ Enteral feeding can be interrupted following the administration of sedatives or muscle relaxants, the presence of secondary underlying diseases, and delayed gastric emptying. Besides, it has been reported that the presence of these factors can also increase the risk of developing aspiration pneumonia in these situations. High gastric residual volume was also reported to be potentially present in the critically ill population. In these children, recommendations by the European society for pediatric gastroenterology, hepatology, and nutrition (ESPGHAN) indicate that enteral nutrition can be achieved via transpyloric tube feeding in cases of failure of gastric feeding approaches. ${ }^{3}$

Metabolic complications were reported to occur secondary to conducting enteral feeding in children. However, it has been demonstrated that these complications are not frequently encountered in these settings. On the other hand, evidence shows that pediatric patients with renal, hepatic, or cardiac conditions and patients with certain nutritional requirements should be given adequate attention to achieve better management. Among patients with chronic nutritional imbalance, adequate attention and high-quality care should also be considered to reduce the potential risk of developing refeeding syndrome secondary to the administration of abrupt feeding of high-energy nutritional components. ${ }^{26}$ Increased insulin secretion might also be a potential complication in these settings secondary to the excessive administration of carbohydrates, which will subsequently result in the shifting of potassium, magnesium, and phosphorus into the cells. ${ }^{1}$ Evidence also indicates that the initial calories of volume supply should be maintained at $<75 \%$ than the required levels to prevent the development of arrhythmia, heart failure, and subsequent death, secondary to the presence of hypophosphatemia.

Some of the common complications include gastrointestinal adverse events. These include cramping, bloating, and intestinal discomfort. ${ }^{5,11}$ Besides, evidence also indicates that nausea and vomiting might be associated with the presence of certain physiological factors, constipation, slow gastric emptying, and excessive perfusion rate. On the other hand, aspiration and regurgitation of food can be associated with intolerance of bolus feeds and dislodged tubes. ${ }^{1}$ The presence of microbial contamination, high feed osmolarity, excessive infusion rate, intolerance of bolus feeds, and the excessive intake of dietary components that are not compatible with the current status and functions of the gastrointestinal tract of the affected child are all significant factors that can potentially attribute to the development of diarrhea in these patients. ${ }^{1}$ Two major events have been associated with the development of infection-related complications. The first event includes the development of a wound-site infection, which includes infections that are related to the site of device insertion at the beginning of the feeding process. Some of these infections might be systemic and local septicemia, peristomal abscess, cellulitis, and purulent discharge. ${ }^{27-30}$ Therefore, applying clean dressings in sterile media around the site of device insertion, in addition to the administration of prophylactic antibiotics after and before conducting the feeding plan has been recommended to intervene against the development of these complications. Contamination of the different delivery sets and the administered formulas are mainly attributable to the development of these infections. ${ }^{31}$ However, it should be noted that the etiology behind the development of these bacterial infections is not adequately investigated and comprehended among the different studies in the literature. Estimates show that the prevalence of these bacterial infections is high across the different pediatric settings, with an estimated rate that ranges between 35 $50 \% .32$ Gram-negative bacilli, Streptococci, and coagulase-negative Staphylococci are the most common organisms that were reported to attribute to the development of these bacterial infections. ${ }^{1}$ Therefore, preparing formulas should be conducted in a sterile medium as infections can potentially develop as a result of the repeated usage of food storage containers, reduced awareness of hygiene, and insufficient hand washing. Other approaches that can be potentially effective in the management of complications and reducing the rates of infections are reducing the time of exposure to the different sources of contamination, like reducing the time of exposure after opening the formula and decreasing feeding hang time. ${ }^{33}$

Other common complications that were reported in these settings might also include the development of mechanical complications. Nevertheless, these complications are not usually serious. ${ }^{6}$ Discomfort is the most reported manifestation secondary to these complications, which is attributed to missing or clogging the naso-enteral tube during the process of initiation or termination of the enteral feeding process. ${ }^{1}$ However, this can lead to other serious complications as perforation, which is a life-threatening condition, and therefore, caution should be considered in these situations to intervene against any unfavorable events and enhance the prognosis of the patient. Enterostomy and gastrostomy tubes were also reported to local irritation and similar complications. The presence of enterocutaneous fistula 
after removal, leakage of gastric juice or nutrients, enlarged stoma site as a result of huge wall incision are also factors that have been proposed to the potential development of stoma-related complications. ${ }^{1}$ Another complication that might occur secondary to enteral feeding is the potential adverse reactions that might be attributed to the administration of different drugs and medications. ${ }^{1,8}$ Accordingly, it is recommended that other routes of administration should be considered when drug administration is indicated. Moreover, the administration of slowly-degrading or coated medications should also be avoided in these situations to intervene against the development of these complications. However, in cases when tubal administration is the only available route of administration, the administration of these medications should be conducted in portions. For instance, dissolving the pills in hot water can be performed to mix these pills with gelatin capsules and water before being ready for administration..$^{5}$

\section{CONCLUSION}

Many indications were reported for the modality, and in general, children that usually suffer from severe weight deficit, weight faltering, and growth retardation are indicated to receive enteral nutrition. Different complications were reported, including mechanical, metabolic, infectious, gastrointestinal, and drug-related complications that might lead to worsened prognosis and can significantly impact the long-term outcomes of these patients. Therefore, paying adequate attention should be considered in these cases to prevent the development of these complications, and provide all the necessary procedures to potentially manage the expected adverse events.

\section{Funding: No funding sources}

Conflict of interest: None declared

Ethical approval: Not required

\section{REFERENCES}

1. Braegger C, Decsi T, Dias JA. Practical approach to paediatric enteral nutrition: a comment by the ESPGHAN committee on nutrition. $\mathrm{J}$ Pediatr Gastroenterol Nutri. 2010;51(1):110-22.

2. Sigalet DL, Mackenzie SL, Hameed SM. Enteral nutrition and mucosal immunity: implications for feeding strategies in surgery and trauma. Can J Surg J Canadien De Chirurgie. 2004;47(2):109-16.

3. Broekaert IJ, Falconer J, Bronsky J. The Use of Jejunal Tube Feeding in Children: A Position Paper by the Gastroenterology and Nutrition Committees of the European Society for Paediatric Gastroenterology, Hepatology, and Nutrition 2019. J Pediatr Gastroenterol Nutri. 2019;69(2):239-58.

4. Romano C, van Wynckel M, Hulst J. European Society for Paediatric Gastroenterology, Hepatology and Nutrition Guidelines for the Evaluation and Treatment of Gastrointestinal and Nutritional
Complications in Children With Neurological Impairment. J Pediatr Gastroenterol Nutri. 2017;65(2):242-64.

5. Yi DY. Enteral Nutrition in Pediatric Patients. Pediatr Gastroenterol Hepatol Nutrition. 2018;21(1):12-9.

6. Kleinman RE, Nutrition AAoPCo. Pediatric nutrition handbook. Amer Academy Pediatrics. 2004.

7. Goulet O, Ruemmele F, Lacaille F, Colomb V. Irreversible intestinal failure. J Pediatr Gastroenterol Nutri. 2004;38(3):250-69.

8. Avitzur Y, Courtney-Martin G. Enteral approaches in malabsorption. Best practice res Clin Gastroenterol. 2016;30(2):295-307.

9. Duggan C, Watkins JB, Koletzko B, Walker WA. Nutrition in pediatrics: basic science, clinical applications. PMPH USA, Ltd; 2016;1.

10. Walker WA, Watkins JB, Duggan C. Nutrition in pediatrics: basic science and clinical applications. BC Decker Inc. 2003.

11. Hays T. Special considerations for managing food allergies. J Parenteral Enteral Nutri. 2012;36(1):56s9s.

12. Critch J, Day AS, Otley A, King-Moore C, Teitelbaum JE, Shashidhar H. Use of enteral nutrition for the control of intestinal inflammation in pediatric Crohn disease. J Pediatr Gastroenterol Nutri. 2012;54(2):298-305.

13. De Lucas C, Moreno M, López-Herce J, Ruiz F, Pérez-Palencia M, Carrillo A. Transpyloric enteral nutrition reduces the complication rate and cost in the critically ill child. J Pediatr Gastroenterol Nutri. 2000;30(2):175-80.

14. Heyman MB, Harmatz P, Acree M. Economic and psychologic costs for maternal caregivers of gastrostomy-dependent children. J Pediatri. 2004;145(4):511-6.

15. Axelrod D, Kazmerski K, Iyer K. Pediatric enteral nutrition. J Parenteral Enteral Nutri. 2006;30(1):S216.

16. Tyson JE, Kennedy KA. Minimal enteral nutrition for promoting feeding tolerance and preventing morbidity in parenterally fed infants. The Cochrane Database Systematic Rev. 2000(2):Cd000504.

17. Ohta K, Omura K, Hirano K. The effects of an additive small amount of a low residual diet against total parenteral nutrition-induced gut mucosal barrier. Am j surg. 2003;185(1):79-85.

18. Chen M-C, Chao H-C, Yeh P-J, Lai M-W, Chen CC. Therapeutic Efficacy of Nasoenteric Tube Feeding in Children Needing Enteral Nutrition. Frontiers Pediatri. 2021;9:126.

19. Damore LJ $2^{\text {nd }}$, Andrus CH, Herrmann VM, Wade TP, Kaminski DL, Kaiser GC. Prospective evaluation of a new through-the-scope nasoduodenal enteral feeding tube. Surg Endoscopy. 1997;11(5):460-63.

20. Kwauk ST, Miles D, Pinilla J, Arnold C. A simple method for endoscopic placement of a nasoduodenal feeding tube. Surg Endoscopy. 1996;10(6):680-83. 
21. Zhang Z, Xu X, Ding J, Ni H. Comparison of postpyloric tube feeding and gastric tube feeding in intensive care unit patients: a meta-analysis. Nutrition Clin. 2013;28(3):371-380.

22. Metheny NA, Stewart BJ, McClave SA. Relationship between feeding tube site and respiratory outcomes. J Parenteral Enteral Nutri. 2011;35(3):346-55.

23. McClave SA, Martindale RG, Rice TW, Heyland DK. Feeding the critically ill patient. Critical Care Med. 2014;42(12):2600-10.

24. Sönmez Düzkaya D, Yildiz S. Effect of two different feeding methods on preventing ventilator associated pneumonia in the paediatric intensive care unit (PICU): A randomised controlled study. Australian Crit Care. 2016;29(3):139-45.

25. Martinez EE, Pereira LM, Gura K. Gastric Emptying in Critically Ill Children. J Parenteral Enteral Nutri. 2017;41(7):1100-9.

26. Kraft MD, Btaiche IF, Sacks GS. Review of the refeeding syndrome. Nutri Clin Practice. 2005;20(6):625-33.

27. Dautle MP, Wilkinson TR, Gauderer MW. Isolation and identification of biofilm microorganisms from silicone gastrostomy devices. J Pediatr Surg. 2003;38(2):216-20.

28. Avitsland TL, Kristensen C, Emblem R, Veenstra M, Mala T, Bjørnland K. Percutaneous endoscopic gastrostomy in children: a safe technique with major symptom relief and high parental satisfaction. J Pediatr Gastroenterol Nutri. 2006;43(5):624-8.

29. Kobak GE, McClenathan DT, Schurman SJ. Complications of removing percutaneous endoscopic gastrostomy tubes in children. J Pediatr Gastroenterol Nutri 2000;30(4):404-7.

30. Ségal D, Michaud L, Guimber D, Ganga-Zandzou PS, Turck D, Gottrand F. Late-onset complications of percutaneous endoscopic gastrostomy in children. J Pediatr Gastroenterol Nutri. 2001;33(4):495-500.

31. Bott L, Husson MO, Guimber D. Contamination of gastrostomy feeding systems in children in a homebased enteral nutrition program. J Pediatr Gastroenterol Nutrit. 2001;33(3):266-70.

32. Roy S, Rigal M, Doit C. Bacterial contamination of enteral nutrition in a paediatric hospital. J Hospital Infect. 2005;59(4):311-6.

33. Lafourcade P, Boulestrau H, Arnaud-Battandier F. Is a 24-h cyclic closed enteral feeding system microbiologically safe in geriatric patients? Clin Nutri. 2002;21(4):315-20.

Cite this article as: Alsafadi DM, Alzahrani GS, Alhayek QM, Alghazwi HH, Alzahrani WA, AL Qahtani RS et al. Indications and long-term results of enteral feeding in infancy and childhood. Int $\mathbf{J}$ Community Med Public Health 2022;9:399-403. 